CERN-PPE/93-13

26 January 1993

\title{
Measurement of Differential Cross Sections for Muon-Neutrino Electron Scattering
}

\author{
The CHARM II Collaboration \\ P. Vilain $\left.{ }^{1}\right)$, G. Wilquet ${ }^{1}$ ) \\ Inter-University Institute for High Energies (ULB-VUB), Brussels, Belgium.
}

R. Beyer, W. Flegel, H. Grote, T. Mouthuy ${ }^{2}$ ), H. Øverås, J. Panman, A. Rozanov $^{3}$ ), K. Winter, G. Zacek, V. Zacek

CERN, Geneva, Switzerland.

\begin{abstract}
F.W. Büsser, C. Foos, L. Gerland, T. Layda ${ }^{4}$ ) F. Niebergall, G. Rädel ${ }^{5}$ ), P. Stähelin, T. Voss

II. Institut für Experimentalphysik ${ }^{6}$ ), Universität Hamburg, Hamburg, Germany
\end{abstract}

D. Favart, Gh. Grégoire, E. Knoops ${ }^{7}$ ), V. Lemaître

Université Catholique de Louvain, Louvain-la-Neuve, Belgium

P. Gorbunov, E. Grigoriev, V. Khovansky, A. Maslennikov

Instutute for Theoretical and Experimental Physics, Moscow, USSR

W. Lippich, A. Nathaniel, A. Staude, J. Vogt

Sektion Physik ${ }^{6}$ ) der Universität München, Munich, Germany

A.G. Cocco, A. Ereditato, G. Fiorillo, F. Marchetti-Stasi, V. Palladino, P. Strolin Università di Napli and INFN Sezione de Napoli, Naples, Italy

A. Capone, D. De Pedis, E. Di Capua ${ }^{8}$ ), U. Dore, A. Frenkel-Rambaldi, P.F. Loverre, D. Macina, G. Piredda, R. Santacesaria

Universià 'La Sapienza' and Instituto Nazionale dei Fisica Nucleare (INFN), Rome, Italy

(Submitted to Phys. Lett. B)

\begin{abstract}
We report on a study of the differential cross sections $d \sigma / d y$ for the processes of elastic $\nu_{\mu^{-}}$and $\bar{\nu}_{\mu^{-}}$electron scattering. The data on which this analysis is based were recorded between 1987 and 1990 with the CHARM II detector in the wide band neutrino beam at the CERN-SPS.

For the first time the shapes of these $y$-distributions have been determined in a modelindependent way. A fit to the data yields for the squares of the neutral coupling constants the ratio $g_{\mathrm{R}}^{2} / g_{\mathrm{L}}^{2}=0.60 \pm 0.19$ (stat.) \pm 0.09 (syst.).
\end{abstract}

1) National Foundation for Scientific Research, Belgium

2) now at Centre de Physique des Particules, Faculté de Luminy, Marseille, France

3) On leave of absence form ITEP (Moscow)

4) now at University of California, Santa Cruz, U.S.A.

5) now at DESY, Hamburg, Germany

6) supported by the German Bundesministerium für Forschung und Technologie under contract numbers $05-4 \mathrm{HH} 22 \mathrm{P}$ and $05-4 \mathrm{MU} 12 \mathrm{P}$

7) Inter-University Institute for Nuclear Science, Belgium

8) Dipartimento di Fisica, Università di Ferrara, Italy 
The helicity structure of the neutral current weak interaction can be investigated in a model independent way by studying the angular distribution of elastic neutrino-electron scattering. Because of the small mass of the electron, the scattering angles in the laboratory system are too small for a direct measurement. The angular distribution is, however, reflected in the energy distribution of the recoiling electrons which can be measured in a fine-grained target calorimeter. Therefore the $y$-distribution, where $y$ is the fraction of the incident neutrino energy carried away by the recoiling electron, is equivalent to the angular distribution.

As the neutrino energies are not known on an event-by-event basis, only the convolution of the true $y$-distributions with the energy spectrum of incident neutrinos is accessible to experimental observation. The extraction of a statistically significant $y$-distribution by an unfolding procedure requires a large number of observed neutrino-electron scattering events.

An experiment dedicated to the study of the purely leptonic, neutral current processes of muon-neutrino electron and muon-antineutrino electron scattering has been carried out by the CHARM-II collaboration in the wide band neutrino beam at CERN. Between 1987 and 1990 more than $2000 \nu e$ events have been recorded in each of the two beam modes $\left(\nu_{\mu}, \bar{\nu}_{\mu}\right)$. This unprecedented number of observed events has encouraged us to perform a direct study of the differential cross sections $d \sigma / d y$ for neutrino electron scattering. As a result we give the ratio of the couplings of the $Z^{0}$ to right- and left-handed electrons.

The differential cross section for the elastic scattering of muon-neutrinos off electrons is given by the expression:

$$
\frac{d \sigma}{d y}\left(\nu_{\mu} e, \bar{\nu}_{\mu} e\right)=\frac{G_{F}^{2}}{\pi} 2 m_{e} E_{\nu}\left[g_{\mathrm{L}, \mathrm{R}}^{2}+g_{\mathrm{R}, \mathrm{L}}^{2}(1-y)^{2}\right] .
$$

$g_{\mathrm{L}}$ and $g_{\mathrm{R}}$ denote the left-handed and right-handed electroweak coupling constants of the electron to the $Z^{0}$. Compared to an isotropic scattering on left-handed electrons, the scattering on righthanded electrons is suppressed by a factor $(1-y)^{2}$ because of angular momentum conservation. From the knowledge of the shape of the $y$-distribution alone, the ratio $g_{\mathrm{R}}^{2} / g_{\mathrm{L}}^{2}$ of left- and righthanded couplings can be determined. In contrast to earlier evaluations of quantities related to $g_{\mathrm{R}}$ and $g_{\mathrm{L}}$, neither absolute cross sections [1] nor the ratio of the cross sections for neutrino and antineutrino electron scattering [2] are used in this analysis; hence no neutrino flux measurement is needed.

The CHARM-II detector consisted of a fine grained target calorimeter, with glass as target material and plastic streamer tubes as active elements followed by a muon spectrometer. For details of the detector and its performance see references [3] and [4]. It was exposed to the CERN

Table 1: Neutrino beam composition

\begin{tabular}{cccc} 
Beam & Component & Relative abundance & $\left\langle E_{\nu}\right\rangle[\mathrm{GeV}]$ \\
\hline$\nu$ & $\nu_{\mu}$ & 1 & $23.7 \pm 0.3$ \\
& $\bar{\nu}_{\mu}$ & $0.072 \pm \mathbf{0 . 0 0 4}$ & $19.2 \pm \mathbf{0 . 5}$ \\
& $\nu_{e}$ & $\mathbf{0 . 0 0 8 7} \pm \mathbf{0 . 0 0 0 1 3}$ & $44.0 \pm 2.2$ \\
& $\bar{\nu}_{e}$ & $\mathbf{0 . 0 0 1 7} \pm \mathbf{0 . 0 0 0 0 3}$ & $\mathbf{3 3 . 8} \pm 1.7$ \\
\hline $\bar{\nu}$ & $\nu_{\mu}$ & $\mathbf{0 . 1 3 6} \pm \mathbf{0 . 0 0 7}$ & $\mathbf{2 6 . 3} \pm \mathbf{0 . 5}$ \\
& $\bar{\nu}_{\mu}$ & 1 & $19.1 \pm \mathbf{0 . 2}$ \\
& $\nu_{e}$ & $\mathbf{0 . 0 0 4 3} \pm \mathbf{0 . 0 0 0 0 6}$ & $37.0 \pm 1.9$ \\
& $\bar{\nu}_{e}$ & $\mathbf{0 . 0 0 7 1} \pm \mathbf{0 . 0 0 0 1 1}$ & $36.5 \pm 1.8$ \\
\hline
\end{tabular}

wide band neutrino beam, which was run in two different modes. Focussing either positive or negative particles the dominant neutrino species could be selected. The polarity was changed 
every 2 to 3 days. The mean neutrino energy was about $20 \mathrm{GeV}$. The beam composition is shown in Table 1. Details about the evaluation of these values can be found in reference [1].

The signature of a neutrino electron $(\nu e)$ event in a calorimeter is an electromagnetic shower in the forward direction. Quantitatively this is reflected by the variable $E_{e} \theta_{e}^{2}$ being kinematically limited to $E_{e} \theta_{e}^{2} \leq 2 m_{e}$, where $E_{e}$ and $\theta_{e}$ denote energy and angle of the outgoing electron and $m_{e}$ is the electron mass. For this reason $E_{e} \theta_{e}^{2}$ is a suitable variable to separate $\nu e$ events from a broader background of semileptonic events with dominantly electromagnetic final states.

The data sample used for the analysis described here was obtained as explained previously in [2] except for one change concerning the measurement of the electron energy. While the shower direction $\theta$ is the most important variable for the background separation and thus for the determination of absolute event numbers [2], the present analysis requires, for the determination of $y$-distributions, in addition good energy resolution. To determine the electron energy we used now the pulseheights, representing the charge induced on aluminum pick-up strips on the back of the streamer tubes. The use of this so-called analog information has the advantage of smaller saturation effects and offers at higher energies a better resolution than the information obtained from the digital read-out of the streamer tubes. A correct estimate of the shower energy, however, requires an elaborate calibration procedure, involving an electron test-beam of known energies and a transportation of this calibration throughout the whole calorimeter by cosmic ray muons and electromagnetic showers. A detailed description of the energy determination can be found in [4].

The differential cross sections $F(y)=d \sigma / d y$ were determined from the measured data with a procedure for regularized unfolding [5] which had already been applied to several similar problems in particle physics [6]. In the present analysis it is based on the two-dimensional histogram $g^{\mathrm{DATA}}\left(E_{e}, E_{e} \theta_{e}^{2}\right)$ representing the measured data. This distribution is related to the differential cross section $F(y)$ by an integral equation:

$$
g^{\mathrm{DATA}}\left(E_{e}, E_{e} \theta_{e}^{2}\right)=\iint S\left(E_{\nu}\right) R\left(E_{e}, E_{e} \theta_{e}^{2}\right) F(y) d y d E_{\nu}+B\left(E_{e}, E_{e} \theta_{e}^{2}\right)
$$

where $R\left(E_{e}, E_{e} \theta_{e}^{2}\right)$ represents the resolution function of the experiment, $S\left(E_{\nu}\right)$ denotes the energy spectrum of the beam and $B\left(E_{e}, E_{e} \theta_{e}^{2}\right)$ stands for the background contributions. The unknown of this equation is $F(y)$ which has to be determined from the measured distribution $g^{\text {DATA }}\left(E_{e}, E_{e} \theta_{e}^{2}\right)$, using the resolution, spectra and background functions. The resolution function is given implicitly by means of a set of simulated events. They were generated using the knowledge of the detector response obtained with an exposure of the detector to an electron test beam of known energies [4]. The simulation has been performed including resolutions, acceptances and neutrino spectra, but avoiding the ad-hoc assumption of a model for the $y$-distribution.

Two different classes of background contribute to $B\left(E_{e}, E_{e} \theta_{e}^{2}\right)$. One is due to semileptonic processes with dominantly electromagnetic final states. These are the processes of coherent and diffractive $\pi^{0}$-production, electron neutrino nucleon scattering, and inclusive neutrino reactions. The $\left(E, E_{e} \theta_{e}^{2}\right)$ distributions of such processes have been modelled using either data or Monte Carlo techniques [2]. Their contribution to the measured $\left(E, E_{e} \theta_{e}^{2}\right)$ distributions was evaluated from a fit of the four modelled background distributions to the measured $\left(E_{e}, E_{e} \theta_{e}^{2}\right)$ distributions in the region of $6 \mathrm{MeV} \leq E_{e} \theta_{e}^{2} \leq 72 \mathrm{MeV}$, containing only background events, and extrapolating to the signal region below $4.5 \mathrm{MeV}$.

The second class of background is composed of events produced by neutrinos of the wrong helicity or by electron-type neutrinos scattering on electrons. Their numbers cannot be determined in a straightforward, model independent way, as the cross sections of these processes depend on $\sin ^{2} \Theta_{\mathrm{W}}$. This dependence, however, was found to be negligible compared to the statistical uncertainty (see Table 3). For the subtraction we used: $\sin ^{2} \Theta_{\mathrm{W}}=0.2337$, the most recently published value obtained by the LEP experiments [7]. 
The unfolding procedure uses sets of measured and simulated events as input and reconstructs binned values for the distribution $F(y)$ with a full covariance matrix for the binned values. The variables used are $\sqrt{E_{e}}$ and $\sqrt{E_{e} \theta_{e}^{2}}$ in the kinematical range of $3 \mathrm{GeV} \leq E_{e} \leq 24 \mathrm{GeV}$ and $E_{e} \theta_{e}^{2} \leq 4.5 \mathrm{MeV}$. The latter cut was introduced to remove as many background events as possible without losing signal events.

The unfolded differential cross sections for $\nu_{\mu} e$ and $\bar{\nu}_{\mu} e$ scattering are summarised in Table 2 and shown in Figure 1; statistical errors are given. The $y$-distributions show deviations from isotropy in the $\nu_{\mu} e$ case and from a pure $(1-y)^{2}$ dependence in the $\bar{\nu}_{\mu} e$ case indicating contributions from scattering on right-handed electrons.

Table 2: Differential cross sections $d \sigma / d y$ for $\nu_{\mu} e$ and $\bar{\nu}_{\mu} e$ scattering in arbitrary units.

\begin{tabular}{llll}
$y$-interval & $\nu_{\mu} e \rightarrow \nu_{\mu} e$ & $y$-interval & $\bar{\nu}_{\mu} e \rightarrow \bar{\nu}_{\mu} e$ \\
\hline $0.00-0.27$ & $1.19 \pm 0.25$ & $0.00-0.29$ & $1.37 \pm 0.30$ \\
$0.27-0.52$ & $1.10 \pm 0.29$ & $0.29-0.53$ & $1.12 \pm 0.27$ \\
$0.52-0.78$ & $1.01 \pm 0.39$ & $0.53-0.78$ & $1.03 \pm 0.25$ \\
$0.78-1.00$ & $0.77 \pm 0.37$ & $0.78-1.00$ & $0.46 \pm 0.25$
\end{tabular}

do/dy[a.u.]

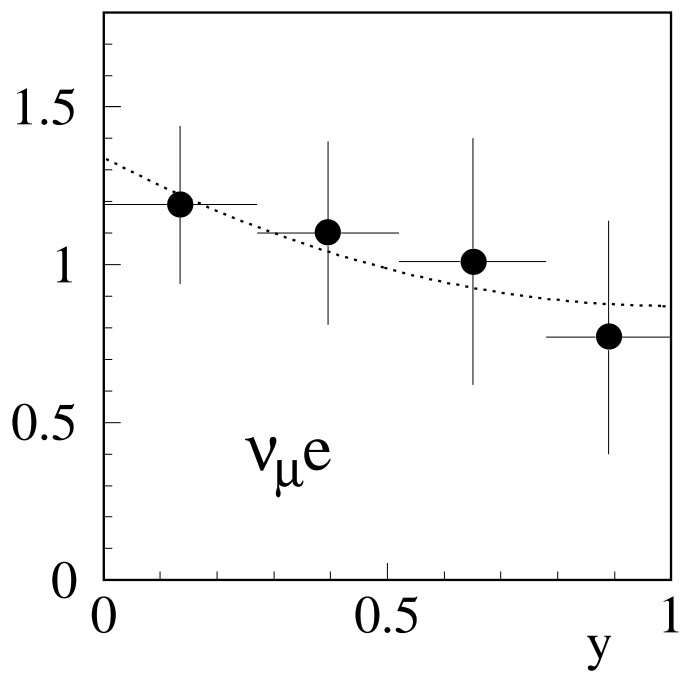

do/dy[a.u.]

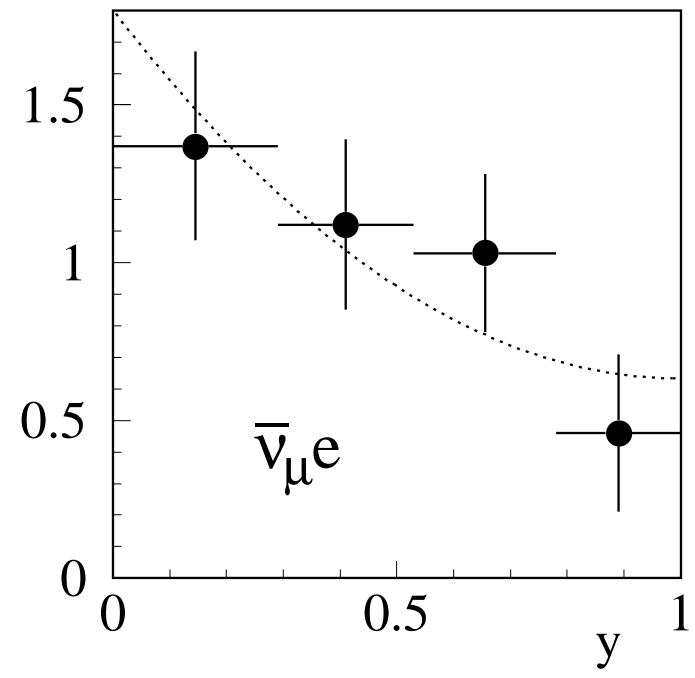

Figure 1: Unfolded differential cross $d \sigma / d y$ sections for $\nu_{\mu} e$ scattering (left), and $\bar{\nu}_{\mu} e$ scattering (right) in arbitrary units. The line overlaid corresponds to the prediction of the Standard Model for a value of the electroweak mixing angle of $\sin ^{2} \Theta_{\mathrm{W}}=0.212$.

A fit of the prediction Eq. (1) to the measured points, taking into account the correlation of the statistical errors, yields for the ratio of the squares of the neutral current coupling constants:

$$
g_{\mathrm{R}}^{2} / g_{\mathrm{L}}^{2}=0.60 \pm 0.19 \text { (stat.) } \pm 0.09 \text { (syst.). }
$$

The contributions to the systematic error, which is dominated by the uncertainty on the energy scale, are listed in Table 3 . This result confirms the existence of a coupling of right-handed electrons to the $Z^{0}$ by three standard deviations and by this non-maximal parity violation in neutral current interactions. It was obtained using only the angular distribution which is the fundamental signature of the handedness of the interaction, disregarding all knowledge on the scale of cross sections. 
Due to the relations of the Standard Model:

$$
g_{\mathrm{L}}=\rho\left(\sin ^{2} \Theta_{\mathrm{W}}-\frac{1}{2}\right) \quad \text { and } \quad g_{\mathrm{R}}=\rho \sin ^{2} \Theta_{\mathrm{W}}
$$

this result is equivalent to a determination of the electroweak mixing angle $\sin ^{2} \Theta_{\mathrm{W}} \cdot \rho$ measures the relative coupling of neutral currents compared to charged currents and plays no role in this kind of analysis, as only the relative determination of right- to left-handed coupling is of interest. Translating equation 3 into a value for $\sin ^{2} \Theta_{\mathrm{W}}$ we obtain:

$$
\sin ^{2} \Theta_{\mathrm{W}}=0.218 \pm 0.021 \text { (stat.) } \pm 0.010 \text { (syst.). }
$$

This result compares well with an electroweak mixing angle

$$
\sin ^{2} \Theta_{\mathrm{W}}=0.212 \pm 0.027 \text { (stat.) } \pm 0.006 \text { (syst.) }
$$

obtained from a direct fit to the shape of the two-dimensional distributions in $E_{e}$ and $E_{e} \theta_{e}^{2}$. For this analysis the same data and analysis method as in our previous publication [1] were used, except that this time no knowledge on the neutrino flux was assumed. This means that in this case we are sensitive mainly to the shape of the $E_{e}$ distribution, which is closely related to the $y$-distribution.

Table 3: Contributions to the systematic error on the ratio $g_{\mathrm{R}}^{2} / g_{\mathrm{L}}^{2}$ and $\sin \Theta_{\mathrm{W}}^{2}$ respectively.

\begin{tabular}{lll}
\hline source & $\delta\left(g_{\mathrm{R}}^{2} / g_{\mathrm{L}}^{2}\right)$ & $\delta\left(\sin ^{2} \Theta_{\mathrm{W}}\right)$ \\
\hline experimental resolutions & 0.005 & 0.0005 \\
energy scale & 0.078 & 0.008 \\
background subtraction $(\nu N)$ & 0.048 & 0.005 \\
background subtraction $(\nu e)$ & 0.003 & 0.0003 \\
$\nu$-beam spectra and composition & 0.019 & 0.002 \\
\hline total & 0.093 & 0.010 \\
\hline
\end{tabular}

The above value of the electroweak mixing angle is in good agreement with our previous measurements [1]. It is the first measurement of the electroweak mixing angle using only the information from the shape of kinematical distributions from neutrino-electron data.

In conclusion: For the first time the shapes of the differential cross sections for elastic muonneutrino electron scattering have been determined in a model independent way. This result has been obtained by unfolding the observed spectra of recoil energies. A fit to the measured points confirms the existence of the coupling of the right-handed electron to the $Z^{0}$ by three standard deviations.

\section{Acknowledgements}

We gratefully acknowledge the work of our many technical collaborators who have contributed to the realization and the operation of the detector. The experiment has been made possible by grants from the Inter-University Institute for Nuclear Sciences (Belgium), CERN (Geneva, Switzerland), the Bundesministerium für Forschung und Technologie (Germany), the Institute of Theoretical and Experimental Physics (Moscow, Russian Federation), and the Istituto Nazionale di Fisica Nucleare (Italy); we gratefully acknowledge their support. We should like to thank the neutrino beam staff and the crew operating the CERN Super Proton Synchrotron for their competent assistance ensuring the excellent performance of the beams. 


\section{References}

[1] CH ARM-II Collab., P. Vilain et al., Phys. Lett. 281 B (1992) 159.

[2] CHARM-II Collab., D. Geiregat et al., Phys. Lett. 259 B (1991) 499.

[3] CHARM-II Collab., K. De Winter et al., Nucl. Instr. \& Meth. A 278 (1989) 670.

[4] CHARM-II Collab., D. Geiregat et al., Calibration and Performance of the CHARM II Detector, Nucl. Inst. \& Meth., (in print).

[5] V. Blobel, DESY 84/118, Hamburg 1984

[6] For example: CHARM Collab., J.V. Allaby et al., Phys. Lett. 213 B (1988) 554 and Phys. Lett. 231 B (1989) 317.

[7] The LEP collaborations: ALEPH, DELPHI, L3 and OPAL, Phys. Lett. B276 (1992) 247. 